\title{
Neutrinoless double beta decay: Experimental challenges
}

\author{
Konstantin Gusev* \\ Joint Institute for Nuclear Research \\ Joliot-Curie, 6 - 141980 Dubna - Russia \\ E-mail: konstantin.gusevejinr.ru
}

The evidence for neutrino flavor oscillations has convincingly shown that neutrino has a finite mass. However, the fundamental question whether neutrino is Majorana or Dirac particle is still unanswered. The only known practical way to probe the Majorana nature of neutrinos experimentally is via the discovery of the neutrinoless double beta $(0 v \beta \beta)$ decay. Moreover, this process violates lepton number conservation. Hence, it is forbidden within the Standard Model of particle physics and, therefore, the discovery of $0 v \beta \beta$ decay will confirm the existence of New Physics. That is the reason why during decades, this search remains worldwide ranked amongst the top research priorities. Many $0 v \beta \beta$ experiments with a target mass around hundred $\mathrm{kg}$ are ongoing and several of next-generation tonne-scale projects are being prepared. The status of some of them is reviewed.

Neutrino Oscillation Workshop (NOW2018)

9 - 16 September, 2018

Rosa Marina (Ostuni, Brindisi, Italy)

\footnotetext{
* Speaker.
} 


\section{Introduction}

In 1936 Maria Göppert-Mayer noted, that in some even-even nuclei the single $\beta$ decay is energetically forbidden whereas the simultaneous but independent $\beta$ decay of two nucleons (socalled double beta decay) is allowed. The main experimental interest is usually focused on two modes of this process: two-neutrino $(2 v \beta \beta)$ and neutrinoless $(0 v \beta \beta)$ double beta decay.

$2 v \beta \beta$ decay is rare (half-life is $\sim 10^{21} \mathrm{yr}$ ) second-order process, in which two neutrinos are emitted, has been observed by now in quite a few isotopes. In the $0 v$ mode the lepton number is not conserved which is forbidden in the Standard Model (SM) of particle physics. So the $0 v \beta \beta$ search is also the search for New Physics beyond the SM.

$\beta \beta$ decay (in both modes) is energetically allowed for 35 nuclei, but its probability strongly depends on the decay energy $Q_{\beta \beta}$. This fact together with the NME value put some constraints on the choice of isotopes and gives the priority to ones with $Q_{\beta \beta}>2 \mathrm{MeV}$. The most used nuclei which fulfill these requirements are ${ }^{48} \mathrm{Ca},{ }^{76} \mathrm{Ge},{ }^{82} \mathrm{Se},{ }^{100} \mathrm{Mo},{ }^{116} \mathrm{Cd},{ }^{130} \mathrm{Te},{ }^{136} \mathrm{Xe}$ and ${ }^{150} \mathrm{Nd}$. The natural abundance of a nucleus is also important, but for all of them, except maybe ${ }^{130} \mathrm{Te}$, the very expensive (and in some cases even not possible) enrichment procedure is required.

In order to reach a half-life sensitivity $>10^{25} \mathrm{yr}$ (level of existing experiments), it is necessary to operate significant target mass and keep the detection efficiency as high as possible. Another essential issue is the background in the region of interest (ROI) close to decay energy. The $0 v \beta \beta$ sensitivity grows linearly with the exposure ( $\mathrm{kg} \mathrm{yr}$ ) as long as there are no background counts in the ROI. So the best experiment should operate in "zero" background mode until the end of data taking. There are two complementary ways to reduce the background: passive (using of radioactively pure materials and placing of an experiment in an underground lab) and active (like pulse shape discrimination (PSD), active veto systems, etc.).

The experimental signature of $0 v \beta \beta$ decay is a peak in the electron sum spectrum at the decay energy. In two neutrino process the full energy is shared with neutrinos which leave the detector unregistered giving the continuous spectrum with the end point close to the decay energy. Thus, $2 v \beta \beta$ decay is unavoidable background source for $0 v \beta \beta$. However this problem can be successfully solved by using a detector with good energy resolution.

$0 v \beta \beta$ decay has not been registered yet, but an experimental limit on its half-life allows to get a limit on effective Majorana neutrino mass $m_{\beta \beta}$ [1]. The main uncertainty in such a transition is coming from nuclear matrix element (NME), which value depends on the nuclear model used for calculation and varies quite a bit [2]. Nevertheless using the most conservative NME numbers it is possible to obtain a reliable limit on $m_{\beta \beta}$.

\section{Overview of the modern experiments}

There are two experimental approaches in $0 v \beta \beta$ decay search. Experiments of the first type so-called "active" source projects are utilizing the idea to use a detector at the same time as a source of double beta decay events. The main advantage of such an experiment is high registration efficiency. It should be mentioned, that most of state-of-art $0 v \beta \beta$ projects are using active source approach. Second way to look for double beta processes is to have an external source (thin film 
source made of $0 v \beta \beta$ isotope is placed between detectors). In this case there is a possibility to measure several isotopes simultaneously and to get full information about electron tracks.

All existing active source experiments can be divided in two groups: projects, which operate with gas or liquid detectors and experiments, where detectors are made from crystals. The serious advantage of the first methodic is relative easiness to get a huge mass of isotope-candidate, whereas crystal detectors usually have very good energy resolution. Today there are many projects aiming to look for $0 v \beta \beta$ decay. It is nearly impossible to mention all of them in a short review. However author fully appreciates the experiments which are not in this list.

\section{1 $0 v \beta \beta$ decay search with liquid detectors}

\subsubsection{EXO-200 and nEXO}

EXO-200 is searching for $0 v \beta \beta$ decay of ${ }^{136} \mathrm{Xe}$ using of a cylindrical time projection chamber (TPC) filled with liquid xenon (LXe) enriched to $80.6 \%{ }^{136} \mathrm{Xe}$ (active mass of xenon is $110 \mathrm{~kg}$ ) [3]. The experiment is located at the Waste Isolation Pilot Plant (WIPP), USA and started data taking in 2010. Latest results of EXO-200 achieved after the electronic upgrade and refilling of LXe performed in 2016 have been recently published [4]. The median $0 v \beta \beta$ half-life sensitivity at the $90 \%$ confidence level (CL) from the full data set has increased two times to $>3.7 \times 10^{25} \mathrm{yr}$ compared with the previous value. No evidence for $0 v \beta \beta$ decay was observed and a lower limit on the $0 v \beta \beta$ half-life for ${ }^{136} \mathrm{Xe}$ was set to $T_{1 / 2}^{0 v}>1.8 \times 10^{25} \mathrm{yr}(90 \% \mathrm{CL}$ ).

The data taking in the upgraded EXO-200 detector is planned to be finished soon giving a way to the tonne scale LXe TPC project - nEXO. This experiment will be located in the SNOLAB, Canada and operate with $5000 \mathrm{~kg}$ of enriched xenon. The expected half-life sensitivity is $\sim 10^{28} \mathrm{yr}$.

\subsubsection{KamLAND-Zen, 800, 2-Zen}

The KamLAND-Zen collaboration also looks for $0 v \beta \beta$ decay of ${ }^{136} \mathrm{Xe}$. The experiment is situated at Kamioka, Japan and exploiting the infrastructure of the KamLAND project. The KamLAND-Zen detector is a $3 \mathrm{~m}$ diameter nylon balloon with 13 tons $\left(380 \mathrm{~kg}\right.$ of $\left.{ }^{136} \mathrm{Xe}\right)$ of Xeloaded liquid scintillator (Xe-LS) immersed in the center of the KamLAND detector (13 m diameter balloon with 1000 ton of liquid scintillator (LS)), used here as an active shield. The scintillation lights are being registered by 1879 photomultiplier tubes (PMTs). The experiment proceeded in two phases from 2011 to 2015. After the completion of first phase Xe-LS was purified. The combination of phase I and phase II (2013-2015, $504 \mathrm{~kg} \mathrm{yr})$ gives a world-best lower half-life limit for ${ }^{136} \mathrm{Xe}$ of $T_{1 / 2}^{0 v}>1.1 \times 10^{26} \mathrm{yr}(90 \% \mathrm{CL})$ [5]. However the reported half-life sensitivity was $>5.6 \times 10^{25} \mathrm{yr}$ and the probability to get a stronger limit was $12 \%$.

Today the next step of KamLAND-Zen project - KamLAND-Zen 800 is under preparation. New inner balloon which fits more Xe-LS $\left(\sim 750 \mathrm{~kg}\right.$ of $\left.{ }^{136} \mathrm{Xe}\right)$ has been installed and filled with dummy LS. Soon, after its replacement by Xe-LS, the data taking is going to be started. The future project KamLAND2-Zen aimed to utilize $\sim 1$ ton of ${ }^{136} \mathrm{Xe}$ and improve the energy resolution.

\subsubsection{SNO+}

SNO+, a kilo-tonne scale liquid scintillator detector, has a reach scientific programm, but the main attention will be given to $0 v \beta \beta$ decay search. The experiment is located in the SNOLAB, 
Canada and consists of a $12 \mathrm{~m}$ diameter acrylic sphere with $\sim 800$ tonnes of LS inside a water bath, viewed by $\sim 10,000$ PMTs [6]. Initially the inner vessel was filled this water. Now pure scintillator phase is ongoing. In 2019 the LS is planned to be loaded with $0.5 \%$ of ${ }^{130} \mathrm{Te}$ which is equal to $1300 \mathrm{~kg}$ of this $0 v \beta \beta$ candidate. $\mathrm{SNO}+$ is willing to reach the half-life sensitivity for ${ }^{130} \mathrm{Te}>1.9 \times 10^{26} \mathrm{yr}$ after 5 years of data taking.

\section{$2.20 v \beta \beta$ decay search with crystals: bolometers}

\subsubsection{CUORE and CUPID}

The CUORE experiment, aimed to search for $0 v \beta \beta$ decay of ${ }^{130} \mathrm{Te}$, uses cryogenic bolometer technic. CUORE detector is an array of $9885 \times 5 \times 5 \mathrm{~cm}^{3}{ }^{\text {nat }} \mathrm{TeO}_{2}$ crystals with a mass of $750 \mathrm{~g}$ each, cooled down up to several $\mathrm{mK}$. The active mass is $742 \mathrm{~kg}$ where $206 \mathrm{~kg}$ is the mass of ${ }^{130} \mathrm{Te}$. After only 2 months of operation $86.3 \mathrm{~kg}$ yr of data were collected. No events of $0 \mathrm{v} \beta \beta$ decay were found and a lower limit $T_{1 / 2}^{0 v}>1.3 \times 10^{25} \mathrm{yr}(90 \% \mathrm{CL}$ ) was placed. The median sensitivity was $>7.0 \times 10^{24}$ yr. Combining this result with obtained by two earlier experiments, Cuoricino and CUORE-0, the most stringent limit for this isotope of $T_{1 / 2}^{0 v}>1.5 \times 10^{25} \mathrm{yr}$ (90\% CL) up to date was set [7]. After 5 years of data taking CUORE plans to overpass the half-life sensitivity of $10^{26} \mathrm{yr}$.

As it was shown by CUORE the main source of background for this experiment is alfa contamination. To get rid of this problem by identifying of particle types the next stage of experiment - CUPID (CUORE Upgrade with Particle IDentification) is being designed. The new promising detector technology - scintillating bolometers was proposed and proven in parallel for $\mathrm{Zn}^{82} \mathrm{Se}$ (CUPID-0) and $\mathrm{Li}_{2}^{100} \mathrm{MoO}_{4}$ (CUPID-Mo) crystals. So it is very well possible that the CUPID project will switch to $0 v \beta \beta$ decay search of ${ }^{82} \mathrm{Se}$ or ${ }^{100} \mathrm{Mo}$ instead of ${ }^{130} \mathrm{Te}$. In any case the mission of the future experiment is to have a sensitivity higher than $10^{27} \mathrm{yr}$.

\section{$2.30 v \beta \beta$ decay search with crystals: Ge detectors}

Historically ${ }^{76} \mathrm{Ge}$ is one of the most famous isotope for double beta search, probably mainly thanks to a claim for discovery of $0 v \beta \beta$ decay reported by part of the Heidelberg-Moscow collaboration, which stayed unchecked for more than 10 years. However there are clear advantages of using Ge detectors enriched in ${ }^{76} \mathrm{Ge}$ in this field, such as high radiopurity of detector-grade germanium and excellent energy resolution and pulse shape discrimination capability of Ge detectors.

\subsubsection{MAJORANA DEMONSTRATOR}

The MAJORANA DEMONSTRATOR is an array of enriched Ge detectors, mounted in a "traditional" vacuum cryostats made from high purity electroformed $\mathrm{Cu}$ [8]. The MAJORANA collaboration performed a very careful assay of construction materials surrounding the detectors and developed low noise read out electronics that helped to get an effective PSD and a low energy threshold. The total mass of enriched Ge detectors is $\sim 30 \mathrm{~kg}$. The experiment started data taking at Sanford Underground Research Facility (SURF), USA in 2015. The lower limit of $T_{1 / 2}^{0 v}>1.9 \times$ $10^{25} \mathrm{yr}(90 \% \mathrm{CL}$ ) was set from the first data release (exposure of $\sim 10 \mathrm{~kg} \mathrm{yr}$ ). The best energy resolution amongst all $0 v \beta \beta$ experiments of $2.5 \mathrm{keV}$ FWHM at $Q_{\beta \beta}$ of ${ }^{76} \mathrm{Ge}(2039 \mathrm{keV})$ was achieved [9]. The collaboration kept taking data and the latest preliminary half-life sensitivity of $4.8 \times 10^{25} \mathrm{yr}(90 \% \mathrm{CL})$, presented at Neutrino 2018, was derived from $26 \mathrm{~kg} \mathrm{yr}$ of data in total. In 
2019 the MAJORANA DEMONSTRATOR is going to be upgraded to improve channel reliability and background, and then reach 50-70 $\mathrm{kg}$ yr of exposure with sensitivity in the range of $10^{26} \mathrm{yr}$.

\subsubsection{GERDA}

The GERDA experiment is also searching for $0 v \beta \beta$ decay of ${ }^{76} \mathrm{Ge}$, but using the innovative approach, when bare germanium detectors are directly immersed in liquid argon (LAr). After the successful completion of the first phase of the experiment the second phase of GERDA has been started. In GERDA Phase II detector mass is doubled by adding novel Ge detectors with improved energy resolution and pulse shape discrimination capability. Total ${ }^{76} \mathrm{Ge}$ mass became about $35 \mathrm{~kg}$. Moreover, in Phase II the LAr is instrumented to readout liquid argon scintillations for vetoing background events [10]. As the result, the background level is reduced down to the unprecedented value of $10^{-3}$ counts $\mathrm{keV}^{-1} \mathrm{~kg}^{-1} \mathrm{yr}^{-1}$. Thus an average background less than one count expected in the ROI up to the design exposure of $100 \mathrm{~kg}$ yr. This implies that GERDA is the first backgroundfree $0 v \beta \beta$ experiment. After the first data release $(\sim 11 \mathrm{~kg} \mathrm{yr}$ of exposure in Phase II $+\sim 24 \mathrm{~kg} \mathrm{yr}$ from Phase I) the collaboration set a lower limit of $T_{1 / 2}^{0 v}>5.3 \times 10^{25} \mathrm{yr}$ at the median half-life sensitivity $4.0 \times 10^{25} \mathrm{yr}$ (both at $90 \% \mathrm{CL}$ ) [11]. When the Phase II exposure has been doubled (in 2017) a $90 \%$ CL limit became $T_{1 / 2}^{0 v}>8.0 \times 10^{25} \mathrm{yr}$ at the world leading at that time sensitivity of $5.8 \times 10^{25} \mathrm{yr}(90 \% \mathrm{CL})$ [12]. The unique background index if normalized to energy resolution was confirmed with more than threefold exposure compared with the previous release. The latest GERDA results from $>80 \mathrm{~kg}$ yr of data in total were presented at Neutrino 2018. The sensitivity of the experiment overcame $10^{26} \mathrm{yr}$ (preliminary) first time ever. In 2018 the collaboration performed an upgrade in order to increase the mass of ${ }^{76} \mathrm{Ge}$ by adding of recently produced novel enriched Ge detectors, show the possibility to improve the background index and prove the robustness and reproducibility of the GERDA approach. Test of these novel diodes so-called "inverted coaxials" is particularly important since detectors of this type are first candidates for tonne-scale Ge project LEGEND. The successful upgrade included: new LAr veto system, installation of 5 inverted coaxial detectors enriched in ${ }^{76} \mathrm{Ge}$ (total increase of isotope mass $\sim 6 \mathrm{~kg}$ ), exchange of all detector cables and repair of broken electronic channels. Now GERDA is continuing data taking until the designed exposure of $100 \mathrm{~kg} \mathrm{yr}$ is achieved, which is planned for the end of 2019. At that time the half-life sensitivity will be above $1.4 \times 10^{26} \mathrm{yr}$.

\subsubsection{LEGEND}

Based on the experience and success of the GERDA and MAJORANA collaborations, the next-generation project LEGEND is being designed and prepared. The experiment is foreseen to proceed in at least two phases. The first phase (LEGEND-200) plans to operate up to $200 \mathrm{~kg}$ of enriched Ge detectors at the background level of $\sim 0.6$ counts FWHM ${ }^{-1} \mathrm{t}^{-1} \mathrm{yr}^{-1}$ and reach the sensitivity of $10^{27} \mathrm{yr}$. The ultimate goal of LEGEND-1000 is to utilize 1 ton of ${ }^{76} \mathrm{Ge}$ at $\sim$ 0.1 counts $\mathrm{FWHM}^{-1} \mathrm{t}^{-1} \mathrm{yr}^{-1}$ and overpass $10^{28} \mathrm{yr}$.

\section{Summary}

Search for $0 v \beta \beta$ decay is a crucial experimental challenge for many years, not only because it is so far a unique possibility to answer the question about neutrino nature, but also that maybe 
opens a way to look for New Physics. Its importance is additionally confirmed by number of the experiments which are ongoing or in preparation. The next generation projects, aimed to investigate full neutrino mass range predicted for the inverse mass hierarchy, are titanic efforts - they have to operate with tonnes of material at "zero" background. However the success of ongoing experiments provides positive expectation that at least a few tonne-scale projects will be realized. It is worth to mention that, as it was estimated recently, the discovery probability of future experiments is significant even in case of normal ordering [13], and, by sure, the convincing claim for discovery of $0 v \beta \beta$ decay has to be proven with multiple isotopes using different experimental methods.

\section{Acknowledgments}

This paper is the write up of a talk given at the Neutrino Oscillation Workshop 2018 (NOW 2018). The author would like to thank again the NOW 2018 organizers for the kind invitation and the GERDA Collaboration for the designation.

\section{References}

[1] S. Dell'Oro, S. Marcocci and F. Vissani, New expectations and uncertainties on neutrinoless double beta decay, Phys. Rev. D 90 (2014) 033005.

[2] J. Engel and J. Menéndez, Status and future of nuclear matrix elements for neutrinoless double-beta decay: a review, Reports on Progress in Physics 80 (2017) 046301.

[3] M. Auger et al. (EXO-200 collaboration), The EXO-200 detector, part I: detector design and construction, J. Instrum. 7 (2012) P05010.

[4] J. B. Albert et al. (EXO-200 collaboration), Search for Neutrinoless Double-Beta Decay with the Upgraded EXO-200 Detector, Phys. Rev. Lett. 120 (2018) 072701.

[5] A. Gando et al. (KamLAND-Zen collaboration), Search for Majorana Neutrinos Near the Inverted Mass Hierarchy Region with KamLAND-Zen, Phys. Rev. Lett. 117 (2016) 082503.

[6] S. Andringa et al. (SNO+ collaboration), Current Status and Future Prospects of the SNO+ Experiment, Advances in High Energy Physics 20166194250.

[7] C. Alduino et al. (CUORE collaboration), First Results from CUORE: A Search for Lepton Number Violation via $0 \beta \beta$ Decay of ${ }^{130}$ Te, Phys. Rev. Lett. 120 (2018) 132501.

[8] N. Abgrall et al. (MAJORANA Collaboration), The MAJORANA DEMONSTRATOR Neutrinoless Double-Beta Decay Experiment, Advances in High Energy Physics 2014365432.

[9] C. E. Aalseth et al. (MAJORANA Collaboration), Search for Neutrinoless Double- $\beta$ Decay in ${ }^{76}$ Ge with the MAJORANA DEMONSTRATOR, Phys. Rev. Lett. 120 (2018) 132502.

[10] M. Agostini et al. (GERDA Collaboration), Upgrade for Phase II of the GERDA experiment, Eur. Phys. J. C 78 (2018) 388.

[11] M. Agostini et al. (GERDA Collaboration), Background-free search for neutrinoless double- $\beta$ decay of ${ }^{76}$ Ge with GERDA, Nature 544 (2017) 47.

[12] M. Agostini et al. (GERDA Collaboration), Improved Limit on Neutrinoless Double- $\beta$ Decay of ${ }^{76}$ Ge from GERDA Phase II, Phys. Rev. Lett. 120 (2018) 132503.

[13] M. Agostini, G. Benato and J. A. Detwiler, Discovery probability of next-generation neutrinoless double- $\beta$ decay experiments, Phys. Rev. D 96 (2017) 053001. 